\title{
FTIR-based spectroscopic analysis in the identification of clinically aggressive prostate cancer
}

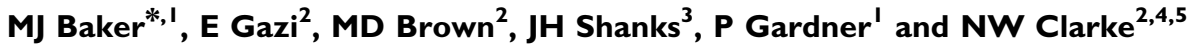 \\ 'Manchester Interdisciplinary Biocentre, Centre for Instrumentation and Analytical Science, School of Chemical Engineering and Analytical Science, The \\ University of Manchester, Manchester, MI 7DN, UK; ${ }^{2}$ Genito Urinary Cancer Research Group, School of Cancer and Imaging Sciences Paterson Institute \\ for Cancer Research, University of Manchester, Wilmslow Road, Manchester, M20 4BX, UK; ${ }^{3}$ Department of Histopathology, Christie Hospital NHS \\ Foundation Trust, Manchester, M20 4BX, UK; ${ }^{4}$ Department of Urology, Christie Hospital NHS Foundation Trust, Manchester, M20 4BX, UK; \\ ${ }^{5}$ Department of Urology, Salford Royal NHS Foundation Trust, Salford, M6 8HD, UK
}

Fourier transform infrared (FTIR) spectroscopy is a vibrational spectroscopic technique that uses infrared radiation to vibrate molecular bonds within the sample that absorbs it. As different samples contain different molecular bonds or different configurations of molecular bonds, FTIR allows us to obtain chemical information on molecules within the sample. Fourier transform infrared microspectroscopy in conjunction with a principal component-discriminant function analysis (PC-DFA) algorithm was applied to the grading of prostate cancer (CaP) tissue specimens. The PC-DFA algorithm is used alongside the established diagnostic measures of Gleason grading and the tumour/node/metastasis system. Principal component-discriminant function analysis improved the sensitivity and specificity of a three-band Gleason score criterion diagnosis previously reported by attaining an overall sensitivity of $92.3 \%$ and specificity of $99.4 \%$. For the first time, we present the use of a two-band criterion showing an association of FTIR-based spectral characteristics with clinically aggressive behaviour in CaP manifest as local and/or distal spread. This paper shows the potential for the use of spectroscopic analysis for the evaluation of the biopotential of $\mathrm{CaP}$ in an accurate and reproducible manner.

British Journal of Cancer (2008) 99, I859- 1866. doi: I0.1038/sj.bjc.6604753 www.bjcancer.com

Published online 4 November 2008

(c) 2008 Cancer Research UK

Keywords: FTIR microspectroscopy; prostate cancer; molecular diagnosis; Gleason grade; PC-DFA; infrared spectroscopy

Prostate cancer $(\mathrm{CaP})$ is the most common cancer and second most common cause of cancer-related deaths of men in the United Kingdom, with 10164 deaths in 2003 (Cancer Research UK, 2006). The diagnosis of $\mathrm{CaP}$ and in particular, the prediction of biopotential in individual patients can be very problematic. Biopotential refers to the ability of the disease to progress to severe cancer. This has led to an inappropriate treatment and pathological upstaging in some cases and an overtreatment of biologically indolent disease in others (Parker et al, 2006).

Confirmation of initial diagnosis involves a biopsy and use of the Gleason grading system for histological analysis. This technique, which is the mainstay for diagnosis and prediction of tumour biopotential, utilises the Gleason grading system (Gleason, 1977) based on tumour morphology, identifying a complex continuum of glandular architecture enabling the grouping of cancer types into five visually distinct grades. This technique, although widely used, has well-recognised flaws. In particular, there are many theoretically low-grade/'low-biopotential' tumours, which show evidence of progression and in addition, there is considerable inter- and intra-observer error in the assignation of the final tumour grade. In a study of 390 patients, identical grades were assigned to only $29.2 \%$ of tumours (Latouf and Saad, 2002),

*Correspondence: Dr MJ Baker; E-mail: M.J.Baker@manchester.ac.uk Received I 3 May 2008; revised 28 September 2008; accepted I October 2008; published online 4 November 2008 and a UK-based study using $81 \mathrm{CaP}$ slides found that on first readings the consensus score was only $78 \%$ (Melia et al, 2006).

The limitation with these diagnostic modalities has led to an interest in the development of spectroscopic analytical techniques for the diagnosis of cancer. Infrared (IR) spectroscopy is a nondestructive method for the analysis of tissues, fluids and cells (Sahu and Mordechai, 2005). Infrared radiation causes vibration of the bonds of the molecules within the sample that absorbs it. The wavelength of the IR radiation absorbed depends on the atoms involved in the bond and the strength of any intermolecular interactions. Therefore, each molecule can have a different spectrum; in essence, the IR spectrum is a fingerprint of the sample. Infrared spectra of biomolecules allow the measurement of complex molecular vibrational modes that contain valuable information on changes occurring due to diseases such as cancer. Infrared spectroscopy is a quick, cost-effective, simpleto-operate, reagent-free technique that requires simple sample preparation.

Fourier transform infrared (FTIR) spectroscopy coupled with advanced computational methods has been used to detect many different forms of cancer from tissue biopsies. These include benign and malignant prostate (Gazi et al, 2003, 2004, 2006), colon (Lasch et al, 2002) and cervical (Wood et al, 2004) tissues, all of which have been evaluated using this technique and with a high rate of diagnostic accuracy. In relation to grading of $\mathrm{CaP}$, the sensitivities and specificities reported earlier have been as high as 78 and $89 \%$, respectively, using a Fourier transform infrared-linear 
discriminant analysis model to predict Gleason scores less than $7(\mathrm{GS}<7)$, equal to $7(\mathrm{GS}=7)$ and greater than $7(\mathrm{GS}>7)$ (Gazi et al, 2006).

This paper discusses the use of FTIR microspectroscopy combined with principal component-discriminant function analysis (PC-DFA) to evaluate formalin-fixed archival CaP tissue based upon a three-band and a two-band Gleason score criteria. The aim of this paper is to determine the possible biochemical changes associated with the progression of $\mathrm{CaP}$ and to observe whether there are characteristic spectroscopic changes between prostate confined and locally invasive tumours.

\section{MATERIALS AND METHODS}

\section{Primary tissue preparation and sampling for FTIR}

With full ethical committee approval, Trent MREC 01/4/061, tissue was collected from 39 consenting patients undergoing transurethral resection of the prostate for bladder outflow obstruction. Forty CaP tissue biopsy specimens from 39 men were obtained as paraffin-embedded blocks (Genito-Urinary Cancer Research Group, Paterson Institute for Cancer Research) from patients with $\mathrm{CaP}$. The tissue was fixed in $4 \%$ formalin for $24 \mathrm{~h}$, then placed onto a Thermoshandon Excelsior processor that passes 20,90 and four $100 \%$ ethanol aliquots through the tissue for an hour each, followed by three changes of xylene for an hour each at a temperature of $40^{\circ} \mathrm{C}$, followed by three changes of paraplast for an hour each at $62^{\circ} \mathrm{C}$. Then the tissue was embedded in a mould of molten wax and cooled on an ice plate for an hour before storage.

Serial sections were collected at $10 \mu \mathrm{m}$ thickness from each

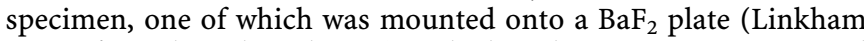
Scientific Ltd, Tadworth, UK) with the adjacent section mounted onto a glass slide and stained with haematoxylin and eosin (H\&E). An experienced histopathologist with a subspecialist interest in genitourinary pathology, including participation in the UK national prostate histopathology external quality assurance scheme, assigned Gleason scores to areas of malignancy identified within the H\&E sections. The complementary sections of the same cancer region were mounted onto $\mathrm{BaF}_{2}$ plates and washed on an orbital mixer with citroclear for $6 \mathrm{~min}$ to remove the paraffin and then acetone at $4{ }^{\circ} \mathrm{C}$ for a further $6 \mathrm{~min}$ before being air-dried for $1 \mathrm{~h}$ under ambient conditions. The anatomical features identified from the H\&E section were used as landmarks to position the IR beam upon the malignant lesions of the unstained adjacent section.

\section{FTIR microspectroscopy}

Fourier transform infrared spectra of Gleason-graded primary prostate tissues were collected in transmission mode using a Nicole Magna system 550 spectrometre equipped with a liquid nitrogen-cooled MCT/A detector and a $\mathrm{KBr}$ beam splitter. The spectrometer is attached to a microscope equipped with a video camera to view optical images ( $\times 150$ magnification) of the sampling area and a programmable computerised $x-y$ stage. An aperture size of $60 \times 60 \mu \mathrm{m}$ was used to collect malignant epithelial cell spectra. Fourier transform infrared spectra represent an average of 512 scans in the mid-IR wavenumber range $750-$ $4000 \mathrm{~cm}^{-1}$ with a spectral resolution of $4 \mathrm{~cm}^{-1}$. Background scans were obtained from a region outside the sample field and ratioed against the sample spectrum. In total, 395 FTIR spectra were obtained from the $40 \mathrm{CaP}$ tissue biopsies from 39 patients with at least five spectra recorded from each patient. The total number of spectra acquired from each patient was dependent on the size of the tumour lesion within the tissue section as well as on the positive identification of tumour glands in the unstained tissue section.

\section{Data processing}

Data processing was carried out immediately after spectral acquisition using OMNIC v.5.1a software. OMNIC software was used to convert the FTIR absorbance spectra into first derivatives (Savitzky-Golay algorithm, nine smoothing points) and second derivatives. The spectral region used was $750.1859-3999.706 \mathrm{~cm}^{-1}$ with the variable $\mathrm{CO}_{2}$ region from 1847.501 to $2809.22 \mathrm{~cm}^{-1}$ removed. This resulted in 1188 spectral data points for analysis. The spectra were then vector normalised in $\mathrm{Matlab}^{\mathrm{TM}}$, to correct for baseline shifts (scattering). Three different pre-processing procedures were used on raw spectra and assessed for optimum discrimination; these steps were (1) vector normalisation (model A), (2) vector normalisation combined with first derivatisation (model B) and (3) vector normalisation combined with second derivatisation (model C). Figure 1 shows raw spectral data and the effect of three pre-processing steps above on an example subset of the FTIR data with the variable $\mathrm{CO}_{2}$ region removed. Matlab coupled with an in-house written software was used to perform PC-DFA. Principal component-discriminant function analysis uses principal component analysis to reduce the dimensionality of the data before DFA. Discriminant function analysis then discriminates between groups on the basis of the resulting PCs and the $a$ priori knowledge of the group memberships that are fed into the DFA algorithm (Baker et al, 2008). Maximising the inter-group variance and minimising the intra-group variance achieve this. The maximum number of discriminant functions available is the number of groups minus one. The optimum number of PCs was determined through an iterative process of chemometric model generation and validation. Discriminant function analysis is a supervised technique and the model is supplied with information about group membership, so any result produced by the model needs to be tested. This testing was carried out by retaining onefifth of the total filtered pre-processed spectra, randomly selected as an independent test set, and then supplying the spectra to the model as a test set and observing where the model places the spectra on a graphical output. Error ellipses with 95 and $90 \%$ confidence are added to the discriminant function plots. This was achieved using error_ellipse.m written by AJ Johnson and obtained from Matlab central file exchange. Covariance matrices were calculated from the discriminant function analysis score matrix for each grouping, where the centroid was defined as the mean of the discriminant function analysis score matrix for each grouping.

\section{RESULTS}

\section{Correlation of FTIR PC-DFA spectral signature with Gleason score}

The FTIR PC-DFA diagnostic model was derived from $40 \mathrm{CaP}$ tissue biopsies. The 40 biopsies correspond to 395 spectra (90 spectra from 10 biopsies for GS $<7,118$ spectra from 11 biopsies for GS $=7$ and 187 spectra from 19 biopsies for GS $>7$ ). Spectra were divided into a training set consisting of 315 spectra (72 spectra from $\mathrm{GS}<7,94$ spectra from $\mathrm{GS}=7$ and 149 spectra from GS $>7$ ) and a randomly chosen test set consisting of a fifth of the total data set of 80 spectra (18 spectra from GS $<7,24$ spectra from $\mathrm{GS}=7$ and 38 spectra from $\mathrm{GS}>7$ ). The $\mathrm{CaP}$ diagnostic model was built on the training set data and it was at this stage that spectroscopic similarities and differences between spectra collected from different Gleason scores were determined by the PC-DFA algorithm, in a supervised manner. The test set was then used to interrogate the model classification of the different spectra.

The Gleason score is the sum of the predominant and highest Gleason grades observed within a biopsy. When the biopsy has only one Gleason grade, this grade is doubled to produce the resulting Gleason score (Gleason, 1977). A three-band Gleason score criterion was used that divided specimens into groups 
A
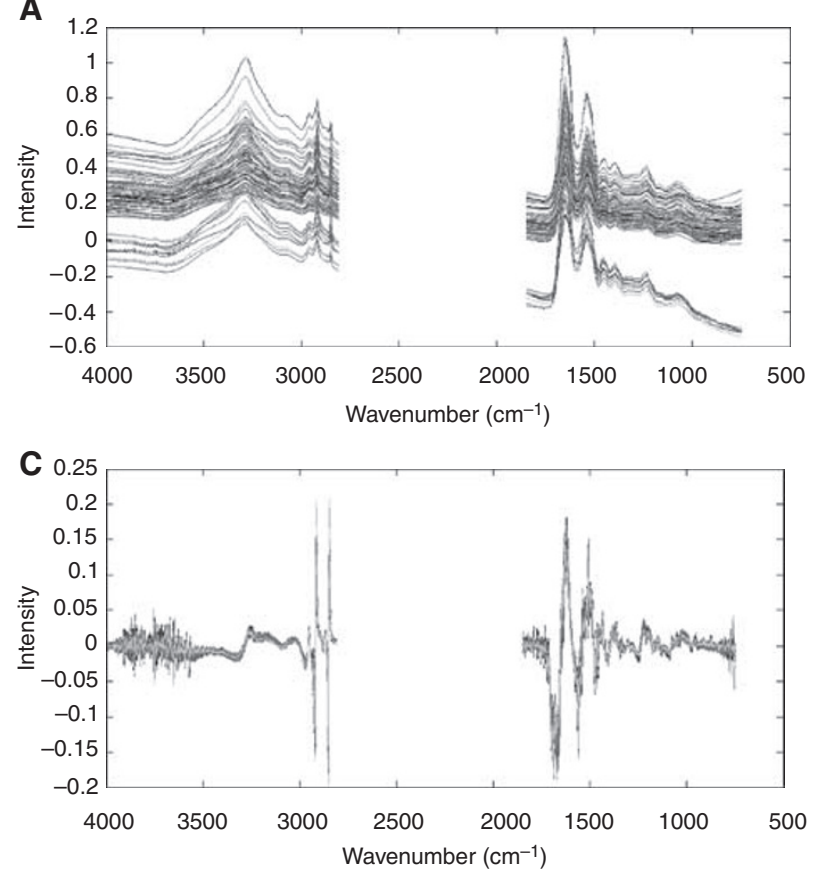

B
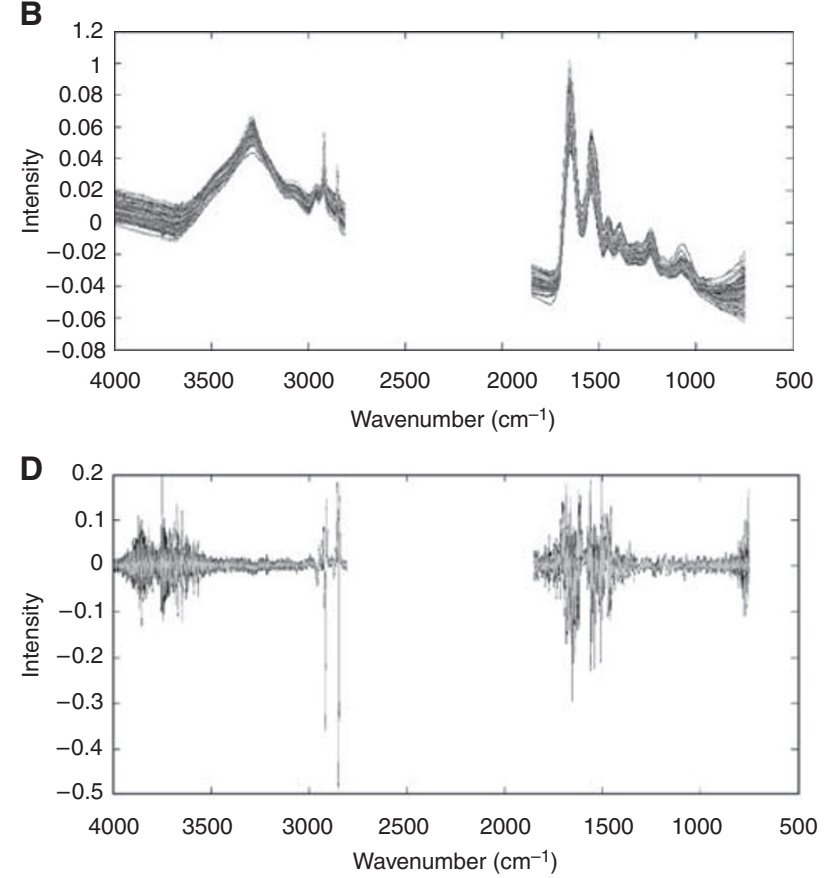

Figure I Raw spectral data $(\mathbf{A})$ and the effect of three pre-processing steps above on an example subset of the FTIR data with the variable $\mathrm{CO}_{2}$ region removed. (B) Vector normalisation (model A), (C) vector normalisation combined with first derivatisation (model B) and (D) vector normalisation combined with second derivatisation (model C).

Table I Sensitivities and specificities observed for less than 7, equal to 7 and greater than 7 Gleason score at 95 and $90 \%$ confidence limits and overall sensitivities and specificities for (i) model A (vector normalised), (ii) model B (vector normalised with first derivative) and (iii) model C (vector normalised with second derivative)

\begin{tabular}{lcccccccc}
\hline Gleason score & \multicolumn{1}{c}{ GS $<\mathbf{7}$} & GS $=\mathbf{7}$ & GS $>\mathbf{7}$ & Overall \\
\hline (i) & & & & & & & & \\
Confidence limit & $95 \%$ & $90 \%$ & $95 \%$ & $90 \%$ & $95 \%$ & $90 \%$ & $95 \%$ & $90 \%$ \\
Sensitivity (\%) & 94.4 & 88.8 & 83.3 & 70.8 & 86.8 & 76.3 & 88.2 & 78.6 \\
Specificity (\%) & 100 & 100 & 98.2 & 98.2 & 100 & 100 & 99.4 & 99.4 \\
(ii) & & & & & & & & \\
Confidence limit & $95 \%$ & $90 \%$ & $95 \%$ & $90 \%$ & $95 \%$ & $90 \%$ & $95 \%$ & $90 \%$ \\
Sensitivity (\%) & 88.9 & 83.3 & 95.8 & 91.7 & 92.1 & 89.5 & 92.3 & 88.2 \\
Specificity (\%) & 98.4 & 100 & 98.2 & 98.2 & 100 & 100 & 98.9 & 99.4 \\
& & & & & & & & \\
(iii) & & & & & & & & \\
Confidence limit & $95 \%$ & $90 \%$ & $95 \%$ & $90 \%$ & $95 \%$ & $90 \%$ & $95 \%$ & $90 \%$ \\
Sensitivity (\%) & 61.1 & 61.1 & 91.7 & 87.5 & 81.6 & 76.2 & 78.1 & 74.9 \\
Specificity (\%) & 98.4 & 100 & 94.6 & 96.4 & 97.6 & 97.6 & 96.9 & 98 \\
\hline
\end{tabular}

corresponding to GS $<7, \mathrm{GS}=7$ and GS $>7$. This method is a more clinically orientated scale in which Gleason scores $<7$ are less aggressive, Gleason $=7$ are of intermediate biopotential and Gleason scores $>7$ are the most likely to progress. This grouping has been shown earlier to be characterised by specific FTIR spectral characteristics (Gazi et al, 2006). Table 1 shows the sensitivity and specificity observed for each of the bands in the criterion at $95 \%$ and stringent $90 \%$ confidence limits for models obtained using three different data analysis pre-processing steps. These models are (A) spectra vector normalised, (B) spectra vector normalised followed by first derivative and (C) spectra vector normalised followed by second derivative. Sensitivity refers to the proportion of people with disease who have a positive test result

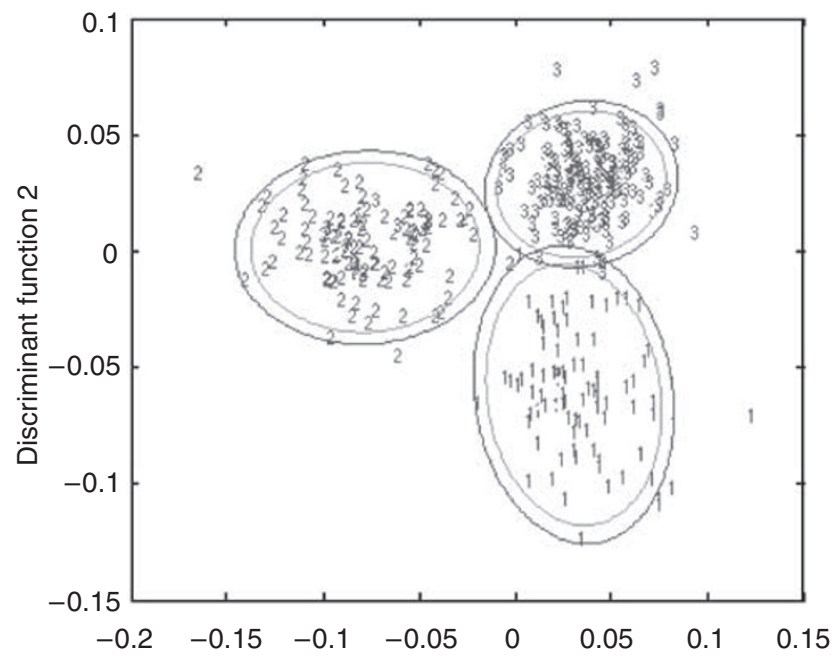

Figure 2 Discriminant function plot showing separation of model B (first derivative vector normalised) based upon the training set (red digits) and test set (blue digits) with 95\% (blue ellipse) and 90\% (green ellipse) confidence limits, where I = Gleason score less than 7, 2 = Gleason score equal to 7 and $3=$ Gleason score greater than 7. DFA - DFI vs DF2.

and specificity refers to the proportion of people without the disease who have a negative test result.

Model B (vector normalised combined with first derivative) achieves the greatest overall sensitivity of $92.3 \%$ for the models at the $95 \%$ confidence limit. The overall sensitivity is the average of the three measurements attained when discriminating the three groups $(\mathrm{GS}<7, \mathrm{GS}=7$ and $\mathrm{GS}>7)$. Figure 2 shows the discriminant function plot for model B showing separation based upon the training set (red digits) and test set (blue digits) with 95\% (blue ellipse) and $90 \%$ (green ellipse) confidence limits, where $1=\mathrm{GS}<7,2=\mathrm{GS}=7$ and $3=\mathrm{GS}>7$. 

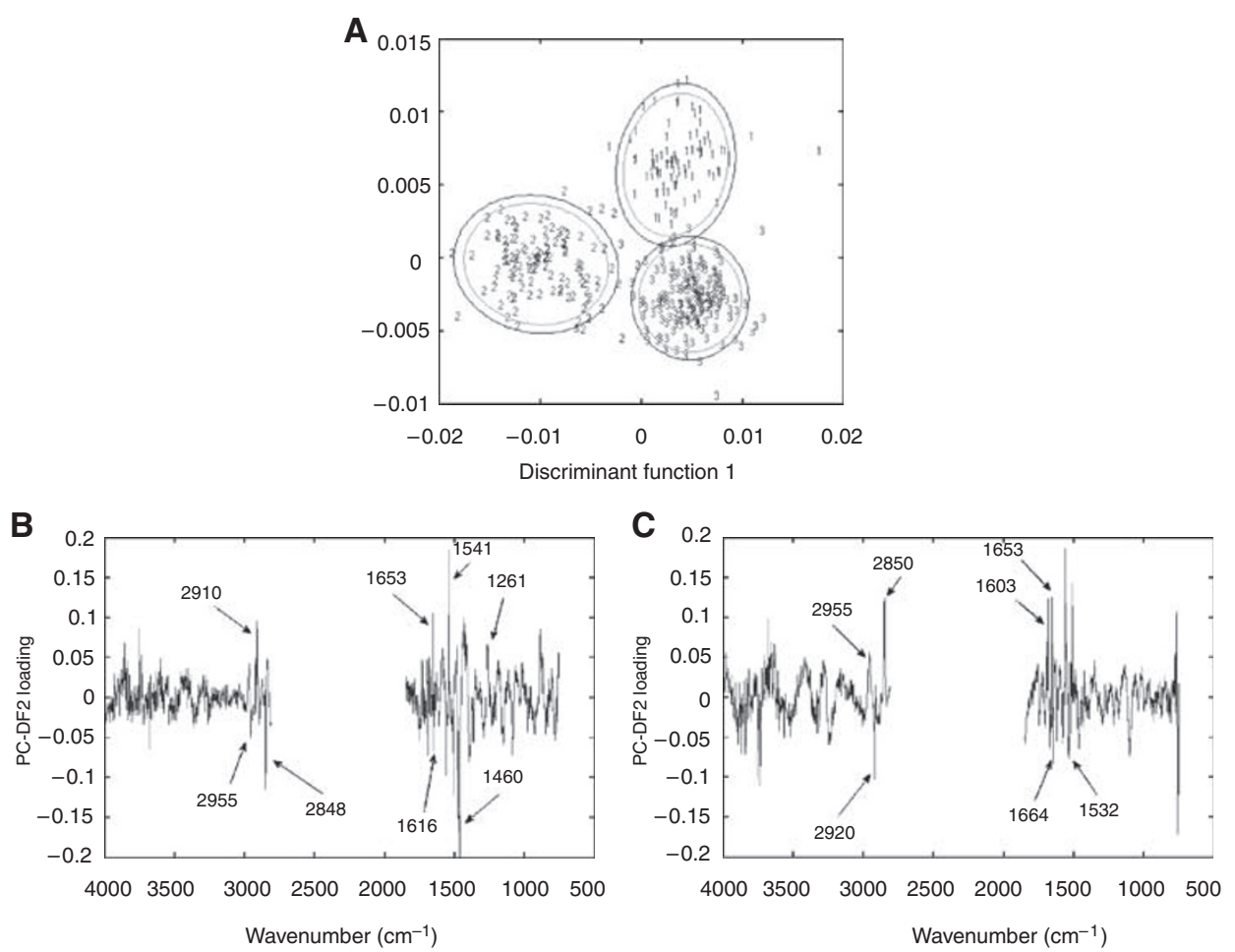

Figure 3 (A) Discriminant function plot showing separation of model A (vector-normalised model) based upon the training set (red digits) and test set (blue digits) with 95\% (blue ellipse) and 90\% (green ellipse) confidence limits, where I=Gleason score less than 7, $2=$ Gleason score equal to 7 and $3=$ Gleason score greater than 7. (B) Loading plots for discriminant function I and (C) discriminant function 2. DFA - DFI vs DF2

Discerning the peaks responsible for discrimination from factor loadings based upon a first derivative spectral model is particularly difficult as the peak in the raw spectrum becomes a point on the baseline in the first derivative. The use of the first derivative in pre-processing spectra removes the additive baseline shift and enhances the chemical information available for discrimination. However, as model A achieves the highest overall specificity of $99.4 \%$ at the $95 \%$ confidence limit from spectra that have only been vector normalised (to remove baseline shifts), model A can be used to indicate which peaks are responsible for discrimination. Figure $3 \mathrm{~A}$ shows the discriminant function plot for model $\mathrm{B}$ based upon training and test set data with confidence ellipses as in Figure 2.Figure $3 \mathrm{~B}$ and $\mathrm{C}$ shows the loading plots for discriminant functions 1 and 2 from which we obtain the spectral peaks responsible for discrimination in model A.

The axes of the discriminant function plot have positive and negative directions (Figure 3 ). The peaks in the positive direction of the $y$ axis of the DF1 loading plots correspond to the spectral peaks that the model is using to discriminate GS $<7$ and GS $>7$ from $\mathrm{GS}=7$, and the peaks in the negative direction are used to discriminate $\mathrm{GS}=7$ from $\mathrm{GS}<7$ and $\mathrm{GS}>7$. The peaks in the positive direction of discriminant function 2 correspond to the spectral peaks used to discriminate GS $<7$ from GS $>7$, and peaks in the negative direction are used to discriminate GS $>7$ from $\mathrm{GS}<7$. Table $2 \mathrm{~A}$ shows the major spectral peaks responsible for the Gleason score discrimination and proposed biomolecular assignments for discriminant function 1 and Table $2 \mathrm{~B}$ for discriminant function 2 .

\section{Correlation of FTIR PC-DFA spectral signature with clinical stage}

The clinical stage is taken from the tumour/node/metastases classification system. For the purpose of this experiment, we are concerned with information from the primary tumour, investigat- ing T1 and T2 tumours (confined to the prostate), T3 tumours (breaching the prostate capsule or invading the seminal vesicle) and T4 tumours (extending beyond the prostate and seminal vesicle to invade local pelvic structures). $\mathrm{N}$ stages are classified as $\mathrm{N} 0, \mathrm{Nx}$ (nodal status unknown) or $\mathrm{N}+$ (nodal status positive) and $M$ stage as M0 or M1. Definitive lymph node staging was not available in T1 and T2 patients: these were designated N0. For the same reasons, most low-stage and -grade tumours were designated Mx.

Clinical staging information was available for 33 out of 39 patients. This corresponds to 347 spectra (191 spectra from 18 biopsies for T1 and T2 and 156 spectra from 15 biopsies for T3 and T4). The spectra were split into a training set consisting of 276 (152 spectra from T1 and T2 and 124 spectra from T3 and T4) spectra and a randomly chosen test set consisting of a fifth of the total data set of 71 spectra ( 39 spectra from T1 and T2 and 32 spectra from T3 and T4). No weighting criteria were applied to the spectra, so each spectrum is of equal importance. Different number of spectra from different groups in the model does not bias the model as we are discriminating upon differences between groups and grouping on similarities within groups.

In our study, a two-band criterion was used that divided specimens into either stage $\mathrm{T} 1$ and $\mathrm{T} 2$ (confined to the prostate) or T3 and T4 (extension outside the prostate). The sensitivity and specificity calculations were based upon spectral assignment to these criteria. If the spectrum was predicted to lie within the correct group then this was counted as a positive prediction. Table 3 shows the sensitivity and specificity observed for each of the bands in the criterion at $95 \%$ and more stringent $90 \%$ confidence limits for (A) model A - spectra vector normalised, (B) model B - spectra vector normalised followed by first derivative and $(\mathrm{C})$ model $\mathrm{C}$ - spectra vector normalised followed by second derivative.

The best-performing model is model $\mathrm{A}$, which attained overall sensitivity and specificity of 91.2 and $93.0 \%$. This model used 62 
Table 2 Major spectral peaks responsible for the Gleason score discrimination and proposed biomolecular assignments for (A) discriminant function I and (B) for discriminant function 2

\begin{tabular}{|c|c|c|}
\hline Direction & $\begin{array}{l}\text { Wavenumber } \\
\quad\left(\mathrm{cm}^{-1}\right)\end{array}$ & Proposed biomolecular assignment \\
\hline \multicolumn{3}{|l|}{ (A) } \\
\hline$+\mathrm{ve}$ & 1063 & C-O stretch, deoxyribose/ribose, DNA, RNA \\
\hline +ve & $|26|$ & Amide III (NH bend (55\%), C-C stretch (19\%), C-N stretch (I5\%), CO bend (I I\%)) \\
\hline +ve & 1265 & Amide III ((NH bend (55\%), C-C stretch (19\%), C-N stretch (I5\%), CO bend (I I\%)) or PO $\mathrm{P}_{2}^{-}$stretch, RNA, DNA \\
\hline +ve & $|54|$ & Amide II of $\alpha$-helical structures ( $\mathrm{NH}$ bend (43\%), C-N stretch (29\%), CO bend (I5\%), C-C stretch (9\%), N-C stretch (8\%)) \\
\hline +ve & 1653 & Amide I of $\alpha$-helical structures or unordered (C-O stretch (76\%), C-N stretch (I4\%), CCN deformation (I0\%)) \\
\hline +ve & 2910 & $\mathrm{C}-\mathrm{H}$ stretch (asymmetric) of $>\mathrm{CH}_{2}$ in fatty acids, lipids, proteins \\
\hline+ ve & 2920 & $\mathrm{C}-\mathrm{H}$ stretch (asymmetric) of $>\mathrm{CH}_{2}$ in fatty acids, lipids, proteins \\
\hline +ve & 2966 & $\mathrm{C}-\mathrm{H}$ stretch (asymmetric) of $\mathrm{CH}_{3}$ in fatty acids, lipids, proteins \\
\hline$-v e$ & 1086 & $\begin{array}{l}\mathrm{C}-\mathrm{O}, \mathrm{C}-\mathrm{C} \text { stretches, } \mathrm{C}-\mathrm{O}-\mathrm{H}, \mathrm{C}-\mathrm{O}-\mathrm{C} \text { deformation of carbohydrates or } \mathrm{PO}_{2}^{-} \text {symmetric stretch of phosphodiester group in nucleic acids } \\
\text { and phospholipids }\end{array}$ \\
\hline$-v e$ & || $5 \mid$ & $\mathrm{C}-\mathrm{O}, \mathrm{C}-\mathrm{C}$ stretch, $\mathrm{C}-\mathrm{O}-\mathrm{H}, \mathrm{C}-\mathrm{O}-\mathrm{C}$ deformation of carbohydrates or $\mathrm{C}-\mathrm{OH}$ stretch of serine, threonine, tyrosine in cell proteins \\
\hline$-v e$ & 1460 & $\mathrm{CH}_{3}$ antisymmetric bend \\
\hline$-v e$ & 1473 & $\mathrm{CH}_{2}$ scissoring \\
\hline- ve & 1616 & Amide I of aggregated strand protein structures (C-O stretch (76\%), C-N stretch (I4\%), CCN deformation (I0\%)) \\
\hline$-v e$ & 1650 & Amide I of $\alpha$-helical protein structures (C-O stretch (76\%), C-N stretch (I4\%), CCN deformation (I0\%)) \\
\hline$-v e$ & 1686 & Amide I of antiparallel $\beta$-sheet/aggregated strand protein structures (C-O stretch (76\%), C-N stretch (I4\%), CCN deformation (I0\%)) \\
\hline$-v e$ & 2848 & $\mathrm{C}-\mathrm{H}$ symmetric stretch of $>\mathrm{CH}_{2}$ in fatty acids, lipids and proteins \\
\hline$-v e$ & 2895 & $\mathrm{C}-\mathrm{H}$ stretch of $\mathrm{C}-\mathrm{H}$ in methine groups \\
\hline$-v e$ & 2955 & $\mathrm{C}-\mathrm{H}$ asymmetric stretch of $-\mathrm{CH}_{3}$ in fatty acids, lipids and proteins \\
\hline
\end{tabular}

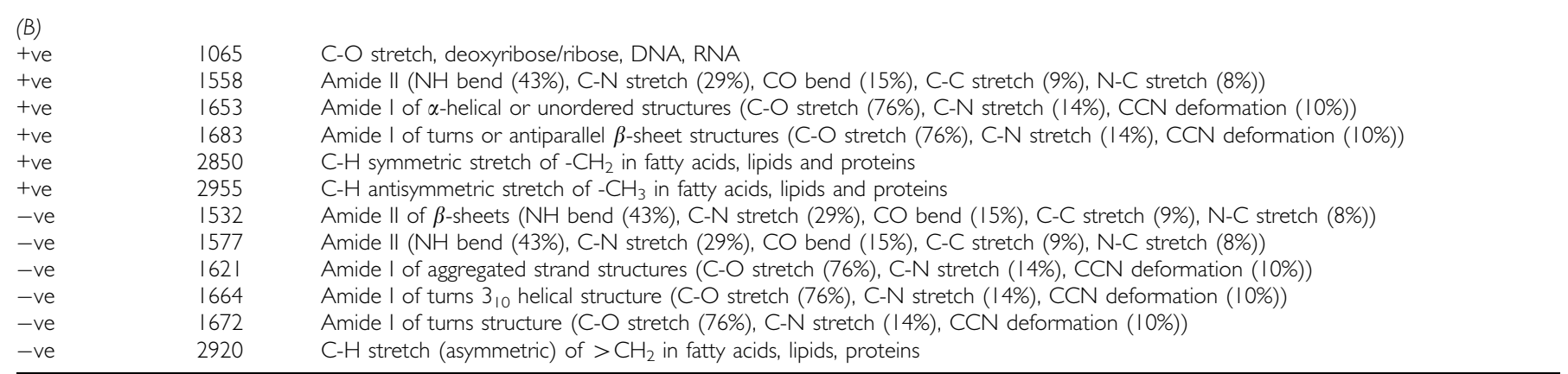

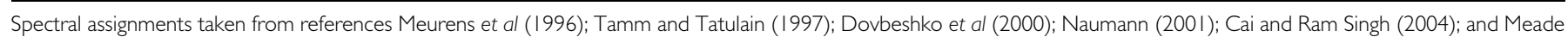
et al (2007).

Table 3 Sensitivities and specificities observed for TI and T2 and T3 and T4 at 95 and 90\% confidence limits and overall sensitivities and specificities for (A) vector normalised, (B) vector normalised with first derivative and (C) vector normalised with second derivative

\begin{tabular}{lrrrrrr}
\hline Clinical stage & \multicolumn{2}{c}{ T I and T2 } & T3 and T4 & \multicolumn{2}{c}{ Overall } \\
\hline (A) & & & & & & \\
Confidence limit & $95 \%$ & $90 \%$ & $95 \%$ & $90 \%$ & $95 \%$ & $90 \%$ \\
Sensitivity (\%) & 94.9 & 92.3 & 87.5 & 84.4 & 91.2 & 88.4 \\
Specificity (\%) & 91 & 91 & 94.9 & 97.4 & 93.0 & 94.2 \\
& & & & & & \\
(B) & & & & & & \\
Confidence limit & $95 \%$ & $90 \%$ & $95 \%$ & $90 \%$ & $95 \%$ & $90 \%$ \\
Sensitivity (\%) & 92.3 & 89.7 & 84.4 & 78.1 & 88.4 & 83.9 \\
Specificity (\%) & 87.5 & 90.6 & 97.4 & 97.4 & 92.5 & 94.0 \\
& & & & & & \\
(C) & & & & & & \\
Confidence limit & $95 \%$ & $90 \%$ & $95 \%$ & $90 \%$ & $95 \%$ & $90 \%$ \\
Sensitivity (\%) & 92.3 & 89.7 & 84.4 & 81.3 & 88.4 & 85.5 \\
Specificity (\%) & 84.4 & 84.4 & 92.3 & 92.3 & 88.4 & 88.4 \\
\hline
\end{tabular}

PCs and one discriminant function. Figure 4A shows the discriminant function plot based upon the training set (red digits) and test set (blue digits) with 95\% (blue ellipse) and 90\% (green ellipse) confidence limits, where $1=\mathrm{T} 1$ and $\mathrm{T} 2$, and $2=\mathrm{T} 3$ and $\mathrm{T} 4$.
Figure 4B shows the loading plots for discriminant function 1, from which it is possible to obtain the peaks responsible for discrimination. As the model has only two groups, only one discriminant function is sufficient for the separation.

The peaks in the positive direction of discriminant function 1 are used to discriminate $\mathrm{T} 3$ and $\mathrm{T} 4$ from $\mathrm{T} 1$ and $\mathrm{T} 2$, and the peaks in the negative direction are used to discriminate $\mathrm{T} 1$ and $\mathrm{T} 2$ from T3 and T4. Table 4 shows the major spectral peaks and possible biomolecular assignments from discriminant function 1 that are used to discriminate the two-band criteria.

\section{DISCUSSION}

\section{Correlation of FTIR PC-DFA spectral signature with Gleason score}

Gleason grading of CaP biopsies is subject to both intra- and interobserver variability, which reduces any prognostic value arising from the grading system (Latouf and Saad, 2002). Fourier transform infrared vibrational spectroscopy determines the chemical profile of a specimen by measuring the entire chemical spectrum by means of absorbance of specific wavenumbers of IR light, which relate to specific chemical bonds. These specific absorbencies are due to bond vibrations, which correlate with individual chemical groups. Interrogation and cross correlation of these enable the acquisition of information detailing the chemical 

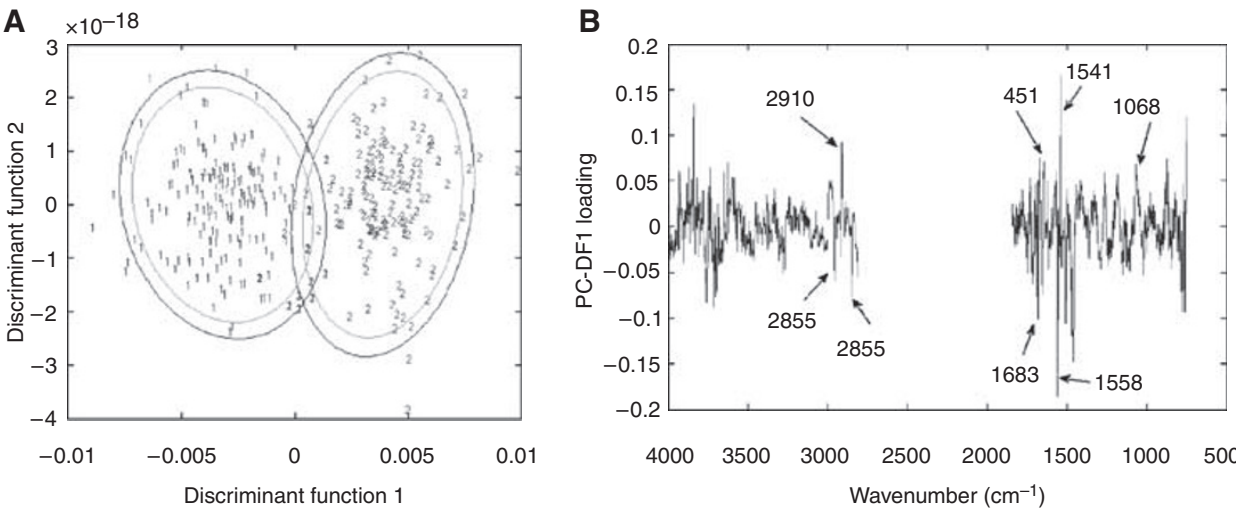

Figure 4 (A) Discriminant function plot showing separation based on model A (vector-normalised model) upon the training set (red digits) and test set (blue digits) with 95\% (blue ellipse) and 90\% (green ellipse) confidence limits, where I=TI and T2 and 2=T3 and T4, and (B) loading plots for discriminant function I. DFA - DFI vs DF2.

Table 4 Major spectral peaks responsible for the 2-band discrimination and proposed biomolecular assignments for discriminant function I

Direction Wavenumber $\left(\mathrm{cm}^{-1}\right)$ Proposed biomolecular assignment

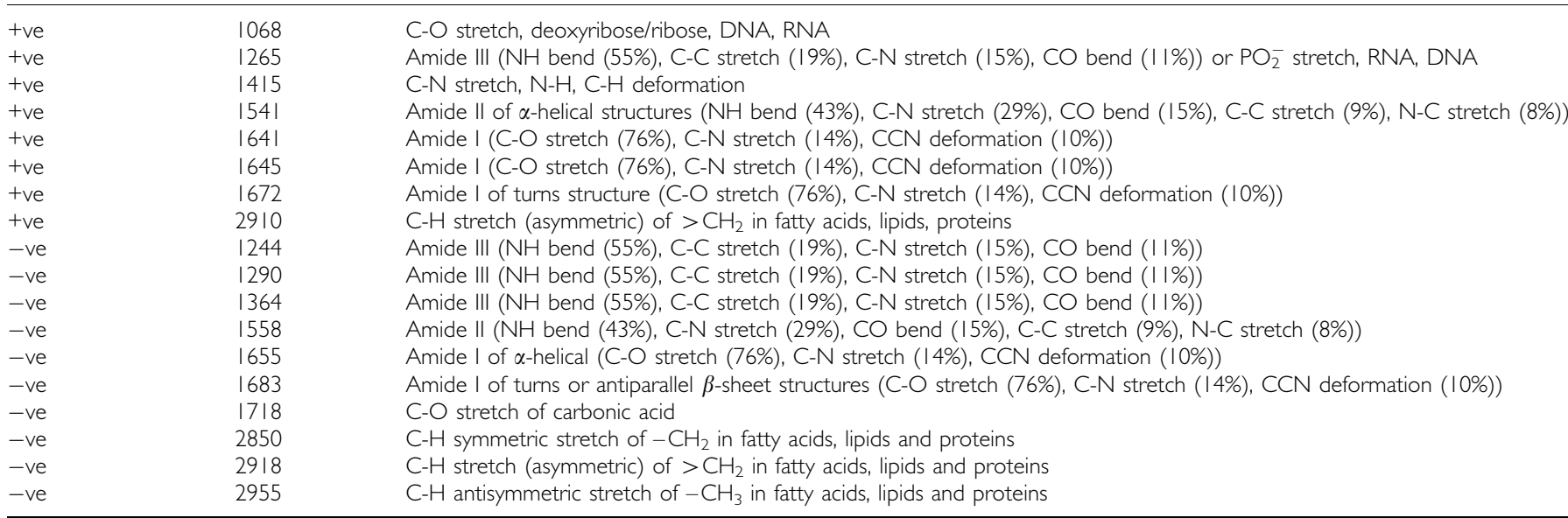

Spectral assignments taken from references Meurens et al (1996); Tamm and Tatulain (1997); Dovbeshko et al (2000); Naumann (200 I); Cai and Ram Singh (2004); and Meade et al (2007).

constituents of local area of tissue within a biopsy (Diem et al, 2004).

To reduce the variability in Gleason grades between specimens, we graded our specimens using a single histopathologist to assign Gleason scores to CaP tissue sections. Latouf and Saad (2002) reported that there was only $29.2 \%$ agreement in the assignment of Gleason score to biopsy and radical prostatectomy samples when assessment was made by multiple pathologists. However, the same study showed that when samples were assessed by a single pathologist, the agreement rose to $48.7 \%$. They concluded that assessment by the same pathologist reduces the discrepancy in the Gleason grading of prostate biopsy.

Although Gleason grading is a useful clinical method, there are clear difficulties with inter-observer variation. Additional diagnostic modalities may provide an important adjunct to diagnosis and, in particular, to the specific identification of tumours with aggressive biopotential. We have shown in earlier studies (Gazi et al, 2006) that chemometric analysis using vibrational spectroscopy is a viable tool for assessing paraffin-embedded formalinfixed $\mathrm{CaP}$ biopsies. This study has developed the technique still further, resulting in a significant improvement in the overall sensitivity and specificity. This was 73 and $86.3 \%$ in the earlier work (Gazi et al, 2006) as compared with 92.3 and $98.9 \%$ achieved in this study.

One of the limitations with this study and the study by Gazi et al (2006) is that the Gleason grading system was used as the reference standard for the development of the diagnostic algorithms. This methodology is inevitably flawed, as it will tend to incorporate problems inherent in the Gleason system into the new system. However, it was felt to be important to investigate this novel area in this manner to establish proof of principle. This goal has now been achieved, with the data currently presented showing clearly that FTIR-based methods can identify and discriminate between CaPs of different types. A study utilising archival tissue from several different tissue banks, increasing the number of cases, would provide a greater test for the discrimination. Future study will now concentrate upon the development of a spectral grading scale in anticipation that this approach may yield alternative and potentially complementary methods for the assessment of $\mathrm{CaP}$.

One of the promising features of these results was the ability to identify a difference in the tissue characteristics of locally confined and locally invasive tumours. The spectral differences between the Gleason scores (Table 2 and Figure 3 ) occur in the amide I (1600$\left.1700 \mathrm{~cm}^{-1}\right)$, amide II $\left(1510-1580 \mathrm{~cm}^{-1}\right)$ and amide III $(1200-$ 
$1400 \mathrm{~cm}^{-1}$ ) regions of the spectra. This suggests a difference in the protein content of the cancerous tissue as the cancer progresses to more aggressive states. Meurens et al (1996) show the amide I and amide II bands to be among the most significant in relation to breast carcinoma, Fabian et al (2006) utilise the amide II region to differentiate colorectal adenocarcinoma from non-cancerous tissue and Bamberry et al (2004) reported that the major spectral differences between the different cells in cervical cancer tissue predictably occur in the amide I region. The majority of the study in FTIR has focussed upon the ability to discriminate cancerous from non-cancerous tissue. Ductal carcinoma in situ has been differentiated from benign breast tissue, with $93 \%$ classified correctly (Fabian et al, 2006). Colorectal adenocarcinoma tissue (Lasch et al, 2002) has been investigated in this way with an overall classification accuracy of $95 \%$. Both these studies used optimised artificial neural networks to process the spectral data. This study has developed spectroscopic analysis further by utilising a PC-DFA algorithm to identify the difference in tumour grade and to predict the absence of local invasion in CaP biopsies. The prediction of biopotential is of particular importance in $\mathrm{CaP}$, where there is a pressing need to differentiate between the indolent and aggressive forms of the disease. The former represents the majority of cancers diagnosed through screening, whereas the latter is responsible for a large number of male cancer deaths (Albertsen et al, 2005; Parker et al, 2006). The results presented herein suggest that the differing biopotential of cancer may be determined by different protein structures corresponding to changes in the amide I peak in the FTIR spectra. The proposed spectral assignments for the positive direction of discriminant function 1 (the spectral peaks $\mathrm{GS}<7$ and $\mathrm{GS}>7$ from GS $=7$ ) have two spectral loading peaks that can be related to $\alpha$-helical structures, whereas the peaks in the negative direction (the spectral peaks that discriminate $\mathrm{GS}=7$ from $G S<7$ and $G S>7$ ) have amide I spectral peaks that can be related to $\alpha$-helical structure and antiparallel $\beta$-sheets/aggregated strands.

The lipid region also aids the differentiation within discriminant function 1, with spectral loading peaks for both groups illuminating different peaks in the lipid region (Figure 3, Table 2). Differentiation based on lipid characteristics has already been proven useful in breast cancer in the differentiation of benign from malignant breast disease (Fabian et al, 2006). Discriminant function 2 is discriminating $\mathrm{GS}<7$ and $\mathrm{GS}>7$. The $\mathrm{GS}=7$ centroid lies close to the zero point on the discriminant function 2 axis, showing that GS $=7$ has components that are present in both $\mathrm{GS}<7$ and GS $>7$. As the GS $=7$ group is made from Gleason grades 3 and 4 , the interrogation of only one of these grades in the $\mathrm{CaP}$ tissue will provide it with attributes of the GS $<7$ or GS $>7$ group. Using this discrimination to shed light upon the biochemicals responsible, we can stipulate that the GS $<7$ group uses spectral peaks that can be attributed to DNA/RNA and $\alpha$ helical, unordered turns or antiparallel $\beta$-sheet protein structures and the GS $>7$ group uses $\beta$-sheet, aggregated strand, $3_{10}$ helical and turns protein structures. The major peaks in the loadings that could be used as a biomarker to distinguish aggressiveness of cancer occur in discriminant function 2. Peaks at 1653 and $1683 \mathrm{~cm}^{-1}$, which are attributable to amide I peaks (Table 2B), are prominent in the loadings for $\mathrm{GS}<7$ and peaks 2920 and
$1577 \mathrm{~cm}^{-1}$, attributable to amide II and $\mathrm{C}-\mathrm{H}$ stretch asymmetric (Table 2B), are prominent in the loadings for GS $>7$.

\section{Correlation of FTIR PC-DFA spectral signature with clinical stage}

To our knowledge, this is the first time that FTIR spectroscopy has been shown to correlate with the local biopotential of $\mathrm{CaP}$. The importance of the identification of tumour aggression has already been documented, but the data from this study also suggest that it may be possible to facilitate the identification of those patients with T3 and T4 disease from those with disease that is truly localised to the prostate. This is a potentially important finding that may be of particular utility in treatment planning, where pathological upstaging of disease previously thought to be of lower stage is a common problem. The FTIR PC-DFA discrimination shows that there is a valid biochemical difference between the two groups of $\mathrm{T}$ stages. This may be useful not only for interrogation of $\mathrm{CaP}$ tissue, but also in the field of biochemical marker development.

The spectral loading peaks responsible for this important discrimination between the two-band $\mathrm{T}$ stage criteria (Table 3 and Figure 4) again are based in the amide and lipid regions, with T1 and T2 using DNA/RNA and $\alpha$-helical structures and T3 and T4 using $\mathrm{C}-\mathrm{O}$ and $\mathrm{C}-\mathrm{C}$ stretches, $\mathrm{C}-\mathrm{O}-\mathrm{H}$ and $\mathrm{C}-\mathrm{O}-\mathrm{C}$ deformations of carbohydrates and aggregated strands, and $\alpha$-helical and antiparallel $\beta$-sheet protein structures. The major peaks in the loadings that could be used as a biomarker to indicate local aggressiveness are at $1558 \mathrm{~cm}^{-1}$, attributable to amide II (for less aggressive locally) (Table 4), and $1541 \mathrm{~cm}^{-1}$, attributable to amide II of $\alpha$-helical structures (more aggressive locally) (Table 4). As with biochemical grading of CaP biopsies by FTIR, FTIR prediction of biopotential relies on detecting subtle changes in protein conformations relating to individual tumour behaviour.

In conclusion, this study reports the use of FTIR combined with PC-DFA to attain overall sensitivities and specificities of 92.3 and $98.9 \%$, respectively, to predict Gleason scores $<7,=7$ and $>7$. These results represent a significant increase in accuracy when compared with the earlier reports. The results also show for the first time that a two-band criterion-based system identifies characteristics that differentiate between tumours that are clinically confined to the prostate from those that are clinically invasive. These findings have significant potential importance in the development of better techniques for the diagnosis, prognostication and treatment planning in $\mathrm{CaP}$.

\section{ACKNOWLEDGEMENTS}

We acknowledge EPSRC and the AICR for financial support. We gratefully thank Mr Gary Ashton and Miss Caron Abbey (Cancer Research UK, Paterson Institute, Manchester) for the preparation of the histological sections and Dr Alex Henderson for programming.

Supplementary Information accompanies the paper on British Journal of Cancer website (http://www.nature.com/bjc)

\section{REFERENCES}

Albertsen PC, Hanley JA, Fine J (2005) 20-year outcomes following conservative management of clinically localized prostate cancer. JAMA 17: $2095-2101$

Baker MJ, Brown MD, Gazi E, Clarke NW, Vickerman JC, Lockyer NP (2008) Discrimination of prostate cancer cells and non-malignant cells using secondary ion mass spectrometry. Analyst 133: 175-179
Bamberry KR, Wood BR, Quinn MA, McNaughton D (2004) Fourier transform infrared imaging and unsupervised hierarchical clustering applied to cervical biopsies. Aust J Chem 57(12): 1139-1143

Cai S, Ram Singh B (2004) A distinct utility of the Amide III infrared band for secondary structure estimation of aqueous protein solutions using partial least squares methods. Biochemistry 43: $2541-2549$ 
Cancer Stats, Mortality-UK, Cancer Research UK (2006) http://info. cancerresearchuk.org/cancerstats/types/prostate/mortality/?a $=5541$ (26th January 2006)

Diem M, Romeo M, Boydston-White S, Miljkovic M, Matthaus C (2004) A decade of vibrational micro-spectroscopy of human cells and tissue (1994-2004). Analyst 129: $880-885$

Dovbeshko GI, Gridina NY, Kruglova EB, Paschuk OP (2000) FTIR spectroscopy studies of nucleic acid damage. Talanta 53: 233-246

Fabian H, Thi NAN, Eiden M, Lasch P, Schmitt J, Naumann D (2006) Diagnosing benign and malignant lesions in breast tissue sections using IR-microspectroscopy. Biochim Biophys Acta 1758: 874-882

Gazi E, Dwyer J, Gardner P, Ghanbari-Siahkali A, Wade AP, Miyan J, Lockyer NP, Vickerman JC, Clarke NW, Shanks JH, Scott LJ, Hart CA, Brown M (2003) Applications of Fourier transform infrared microspec troscopy in studies of benign prostate and prostate cancer. A pilot study. J Pathol 201: 99-108

Gazi E, Dwyer J, Lockyer N, Gardner P, Vickerman JC, Miyan J, Hart CA Brown M, Shanks JH, Clarke N (2004) The combined application of FTIR microspectroscopy and ToF-SIMS imaging in the study of prostate cancer. Faraday Discuss 126: $41-59$

Gazi E, Baker M, Dwyer J, Lockyer NP, Gardner P, Shanks JH, Reeve RS, Hart CA, Clarke NW, Brown MD (2006) A correlation of FTIR spectra derived from cancer biopsies with Gleason grade and tumour stage. Eur Urol 50: 750 - 761

Gleason DF (1977) In Urological Pathology-The Prostate, Tannenbaum M (ed), The Veteran's Administration Cooperative Urologic Research Group: histologic grading and clinical staging of prostatic carcinorma. Lee and Febiger: Philadelphia, p 171
Lasch P, Haensch W, Lewis N, Kidder LH, Naumann D (2002) Characterization of colorectal adenocarcinoma sections by spatially resolved FT-IR microspectroscopy. Appl Spectrosc 48: 1-10

Latouf JB, Saad F (2002) Gleason score on biopsy: is it reliable for predicting the final grade on pathology? BJU Int 90: 694-699

Meade AD, Lyng FM, Knief P, Byrne HJ (2007) Growth substrate induced functional changes elucidated by FTIR and Raman spectroscopy in in-vitro-cultured human keratinocytes. Anal Bioanal Chem 387B: $1717-1728$

Melia J, Moseley R, Ball RY, Griffths DF, Grigor K, Harnden P, Jarmulowicz M, McWilliam LJ, Montironi R, Waller M, Moss S, Parkinson MC (2006) A UK-based investigation of inter- and intra-observer reproducibility of Gleason grading of prostatic biopsies. Histopathology 48(6): 644-654

Meurens M, Wallon J, Tong J, Noel H, Haot J (1996) Breast cancer detection by Fourier transform infrared spectrometry. Vib Spectrosc 10: $341-346$

Naumann D (2001) FT-infrared and FT-Raman spectroscopy in biomedica research. Appl Spectrosc Rev 36(2\&3): 239-298

Parker C, Muston D, Melia J, Moss S, Dearnley D (2006) A model of the natural history of screen-detected prostate cancer and the effect of radical treatment on overall survival. Br J Cancer 94: 1361-1368

Sahu RK, Mordechai S (2005) Fourier transform infrared spectroscopy in cancer detection. Future Oncol 1(5): 635-647

Tamm LS, Tatulain SA (1997) Infrared spectroscopy of proteins and peptides in lipid bilayers. Q Rev Biophys 30(4): 365-429

Wood BR, Chiriboga L, Yee H, Quinn MA, McNaughton D, Diem M (2004) Fourier transform infrared (FTIR) spectral mapping of the cervical transformation zone, and dysplastic squamous epithelium. Gynaecol Oncol 93: 59-68 